\title{
Multi-sensor Target Tracking Using the Bernoulli Filter
}

\author{
Yong Qin ${ }^{1,}$, Hong $\mathrm{Ma}^{1}$, Li Cheng ${ }^{1,2}$, De Yư ${ }^{1}$, Yang $\mathrm{Li}^{1}$ and Xueqin $\mathrm{Zhou}^{1}$ \\ ${ }^{1}$ School of Electronic Information and Communications, Huazhong University of Science and \\ Technology, Wuhan 430074, China
}

${ }^{2}$ School of Electrical and Information Engineering, Wuhan Institute of Technology, Wuhan 430073, China

a qinyong1208@163.com

Keywords: Bernoulli filter, Multi-sensor, Sequential Monte Carlo.

\begin{abstract}
This paper proposes a novel multi-sensor Bernoulli filter (MSBF) based on the finite set statistics (FISST) for tracking a single target under the presence of detection uncertainty and clutter. The proposed algorithm is an extension of original Bernoulli filter in multi-sensor tracking. First, FISST is used to derive multi-sensor likelihood function of the MSBF, and then the sequential Monte Carlo (SMC) method is applied to implement the MSBF. Eventually, the simulation results are provided to demonstrate the effectiveness of the MSBF.
\end{abstract}

\section{Introduction}

Recently, the random finite set (RFS) theory has attracted extensive attention for target tracking, and lots of tracking approaches based on the RFS have been proposed, e.g. the probability hypothesis density (PHD) filter [1][2], the cardinalized PHD filter (CPHD) [3], the multi-Bernoulli filter (MeMBer) [1][4] and the Bernoulli filter [5][6]. In particular, the Bernoulli filter is an optimal Bayes filter for a single target problem of joint detection and tracking, and this paper considers use the measurements from multi-sensor to track a single target in the presence of detection uncertainty and clutter. From a theoretical point of view the original Bernoulli filter addressed only single-sensor scenarios, and the default multi-sensor Bernoulli filter's approximation is the 'iterated corrector approximation' [6]. Note that it requires two steps that the same as the original Bernoulli filter: prediction and update. One first uses the update step to calculate the measurement-updated Bernoulli RFS for the sensor 1; next using this measurement-updated Bernoulli RFS as a new measurement-predicted Bernoulli RFS, and using the update step for the sensor 2 to calculate a new measurement-updated Bernoulli RFS for first two sensors; then same procedure is applied to the remaining sensors.

However, if there are $k$ sensors in the target tracking system, the first $k-1$ sensors largely affect the measurement-predicted Bernoulli RFS, the final measurement-updated Bernoulli RFS mainly depends on the last $k$ th sensor and the final results of target tracking is calculated by the final measurement-updated Bernoulli RFS. Therefore, the ‘iterated corrector approximation' has a feature: changing the order of sensors will produces different track result, and the performance largely depends on the detection probability of the last sensor. When the detection probability of the last sensor is low, the performance of this approach will be poor and not satisfactory.

In order to solve the problem, this paper proposes a novel multi-sensor Bernoulli filter (MSBF) based on the finite set statistics (FISST) [1] for tracking a single target. The proposed algorithm is an extension of original Bernoulli filter in multi-sensor tracking. First, the FISST is used to derive multi-sensor likelihood function with two sensors, notice that it can be easily extended to other multi-sensor systems, and then the sequential Monte Carlo (SMC) approach is applied to implement the MSBF. The simulation results demonstrate that the MSBF can track a single target effectively, and it appears more accurate performance than the ‘iterated corrector approximation'. 


\section{Bernoulli Filter}

As is well known, the Bernoulli filter has two steps: prediction and update. Suppose that the posterior density $\pi_{k-1}$ at time $k-1$ is a Bernoulli RFS and given by $\pi_{k-1}=\left\{r_{k-1}, p_{k-1}\left(x_{k-1}\right)\right\}$, according to [1, Sec.14.7], the recursion of the Bernoulli filter is as follows:

Prediction:

$$
\begin{aligned}
& r_{k \mid k-1}=p_{R, k}\left(1-r_{k-1}\right)+r_{k-1}\left\langle p_{S, k}, p_{k-1}\right\rangle \\
& p_{k \mid k-1}\left(x_{k}\right)=\frac{p_{R, k}\left(1-r_{k-1}\right)}{r_{k \mid k-1}} f_{R, k}\left(x_{k}\right)+\frac{r_{k-1}}{r_{k \mid k-1}}\left\langle f_{k \mid k-1}\left(x_{k} \mid \cdot\right), p_{S, k}(\cdot) p_{k-1}(\cdot)\right\rangle
\end{aligned}
$$

Update:

$$
\begin{aligned}
& r_{k}=\frac{\left\langle\eta_{k}\left(Y_{k} \mid \cdot\right), p_{k \mid k-1}(\cdot)\right\rangle}{\frac{1-r_{k \mid k-1}}{r_{k \mid k-1}} e^{-\left\langle 1, \kappa_{k}\right\rangle} \kappa_{k}^{Y_{k}}+\left\langle\eta_{k}\left(Y_{k} \mid \cdot\right), p_{k \mid k-1}(\cdot)\right\rangle} \\
& p_{k}\left(x_{k}\right)=\frac{\eta_{k}\left(Y_{k} \mid x_{k}\right), p_{k \mid k-1}\left(x_{k}\right)}{\left\langle\eta_{k}\left(Y_{k} \mid \cdot\right), p_{k \mid k-1}(\cdot)\right\rangle}
\end{aligned}
$$

Where $r_{k}$ is target existence probability and $p_{k}\left(x_{k}\right)$ is the spatial probability density of the target state. $\eta_{k}\left(Y_{k} \mid x_{k}\right)$ denotes the likelihood function for a target originated measurement and $\kappa_{k}$ is the intensity of clutter (further details, see [1][4]).

Notice that the Bernoulli filter described above that assumes a single sensor, according to [6], using the 'iterated corrector approximation' that the Bernoulli filter can be extended to multiple sensors system. The step of this algorithm is: use the measurements and the parameters of first sensor to calculate $\left\{r_{k}^{(1)}, p_{k}^{(1)}\left(x_{k}\right)\right\}$ via Eq. (3) and Eq. (4); use $\left\{r_{k}^{(1)}, p_{k}^{(1)}\left(x_{k}\right)\right\}$ as the prior, and then use the measurements and parameters of second sensor to calculate $\left\{r_{k}^{(2)}, p_{k}^{(2)}\left(x_{k}\right)\right\}$ via Eq. (3) and Eq. (4), and so on until we exhaust all of the sensors. Notice that the 'iterated corrector approximation' has a feature: changing the order of sensors will produces different track performance, and the performance largely depends on the detection probability of the last sensor. When the detection probability of the last sensor is low, the measurements from that sensor may be clutter (no target is detected), and then the algorithm may use clutter to update the Bernoulli RFS, which will produce poor quality and not satisfactory.

\section{The Multi-sensor Bernoulli Filter}

In this section, to solve the problem of the traditional MSBF (the 'iterated corrector approximation'), this paper proposes a novel algorithm for the MSBF based on FISST. In the original Bernoulli filter, it is supposed that one target generates one measurement at each scan at the most. Since there is several sensors in the multi-sensor system, one target can generate several measurements at each scan form the different sensors. Therefore, the measurement model of the proposed MSBF is different from the original Bernoulli filter, and the likelihood function $\eta_{k}\left(Y_{k} \mid x_{k}\right)$ is the main difference between the proposed MSBF with the original Bernoulli filter.

\subsection{The RFS Measurement Model for Multi-sensor}

At time $k$, the collection of measurements of multi-sensor includes detection and clutter from different sensors, and it can be represented as a finite subset $Y_{k}$ of the observation space $\Re \subseteq \mathrm{i}^{n}$. Suppose that there are $n$ sensors, $x_{k}$ represents the target state at time $k$, and the RFS measurements can be modeled as follows: 


$$
\begin{aligned}
Y_{k} & =Y_{k, 1}\left(x_{k}\right) \bigcup\left\llcorner\bigcup Y_{k, n}\left(x_{k}\right)\right. \\
& =\Theta_{k, 1}\left(x_{k}\right) \bigcup W_{k, 1} \bigcup \mathbf{L} \bigcup \Theta_{k, n}\left(x_{k}\right) \bigcup W_{k, n} \\
& =\Theta_{k}\left(x_{k}\right) \bigcup W_{k, 1} \bigcup\left\llcorner\bigcup W_{k, n}\right.
\end{aligned}
$$

Where $Y_{k, 1}\left(x_{k}\right)=\Theta_{k, i}\left(x_{k}\right) \bigcup W_{k, i}, i=1, \mathrm{~L}, n$ is the measurements received by sensor $i, \Theta_{k, i}\left(x_{k}\right)$ denotes the measurement RFS from the target and $W_{k, i}$ is the RFS of clutter from sensor $i$, we assume that $\Theta_{k, i}\left(x_{k}\right)$ and $W_{k, i}$ are independent RFSs. $\Theta_{k}\left(x_{k}\right)$ denotes the RFS of the union of the measurements form all the sensors generated by the target.

The clutter $W_{k, i}$ is modeled as a Poisson RFS with intensity $\kappa_{k, i}$, and the measurement of the target received by each sensor $\Theta_{k, i}\left(x_{k}\right)$ is modeled as a Bernoulli RFS with parameters $\left\{p_{D, k}^{i}\left(x_{k}\right), g_{k, i}\left(\cdot \mid x_{k}\right)\right\}, i=1, \mathrm{~L}, n$, where the probability of target detection for sensor $i$ from a target with state $x_{k}$ is given by $p_{D, k}^{i}\left(x_{k}\right)$, and $g_{k, i}\left(\cdot \mid x_{k}\right), i=1, \mathrm{~L}, n$ denotes the likelihood function of sensor $i$.

\subsection{The Multi-sensor likelihood function}

In this section, we take two sensors system as an example to derive the multi-sensor likelihood function corresponding to the above RFS of measurements model.

If the measurements follow the RFS model in Eq. (5), then the likelihood function that the state $x_{k}$ at time $k$ produces the measurement set $Y_{k}$ is given by Eq. (6).

$$
\begin{aligned}
\eta\left(Y_{k} \mid\right. & \left.x_{k}\right)=\left(1-p_{D, k}^{1}\left(x_{k}\right)\right)\left(1-p_{D, k}^{2}\left(x_{k}\right)\right) K_{1}\left(Y_{k, 1}\right) K_{2}\left(Y_{k, 2}\right) \\
& +\sum_{y_{1} \in Y_{k}} p_{D, k}^{1}\left(x_{k}\right)\left(1-p_{D, k}^{2}\left(x_{k}\right)\right) g_{k, 1}\left(y_{1} \mid x_{k}\right) K_{1}\left(Y_{k, 1}-\left\{y_{1}\right\}\right) K_{2}\left(Y_{k, 2}\right) \\
& +\sum_{y_{2} \in Y_{k}}\left(1-p_{D, k}^{1}\left(x_{k}\right)\right) p_{D, k}^{2}\left(x_{k}\right) g_{k, 2}\left(y_{2} \mid x_{k}\right) K_{1}\left(Y_{k, 1}\right) K_{2}\left(Y_{k, 2}-\left\{y_{2}\right\}\right) \\
& \left.+\sum_{\left\{y_{1}, y_{2}\right\} \in Y_{k}} p_{D, k}^{1}\left(x_{k}\right) p_{D, k}^{2}\left(x_{k}\right) g_{k, 1}\left(y_{1} \mid x_{k}\right) g_{k, 2}\left(y_{2} \mid x_{k}\right)\right) \\
& K_{1}\left(Y_{k, 1}-\left\{y_{1}\right\}\right) K_{2}\left(Y_{k, 2}-\left\{y_{2}\right\}\right)
\end{aligned}
$$

Where $K_{i}\left(y_{k}\right)=e^{-\left\langle 1, \kappa_{k, i}\right\rangle} \kappa_{k, i}^{y_{k}}$ is the probability density of clutter RFS for the sensor $i$. Every term in Eq. (6) has its physical interpretation. There are four groups of terms in the likelihood function of the MSBF. The first group represents none of sensors detected the target, the second and three group denotes only one sensor detected the target, the fourth group indicates two sensors detected the target. Notice that it can be easily extended to other multi-sensor systems, which is similar to the method used in [7]

Proof: First, for the ith sensor, the probability mass of the RFS $\Theta_{k, i}\left(x_{k}\right)$ using the set integral [1, Sec.11.3] and probability density of Bernoulli RFS can be given by

$$
\begin{aligned}
\beta_{k, i}\left(S \mid x_{k}\right)=\int_{Y \subseteq S} \pi_{k, i}\left(Y_{k, i} \mid x_{k}\right) \delta Y \\
=1-p_{D, k}^{i}\left(x_{k}\right)+p_{D, k}^{i}\left(x_{k}\right) \int_{S} g_{k, i}\left(y_{i} \mid x_{k}\right) d y_{i} \\
\quad=1-p_{D, k}^{i}\left(x_{k}\right)+p_{D, k}^{i}\left(x_{k}\right) p_{Y_{k, i}}\left(S \mid x_{k}\right)
\end{aligned}
$$

Where $y_{i}$ denotes the measurements received by the sensor $i,\left\{p_{D, k}^{i}\left(x_{k}\right) \mid, g_{k, i}\left(y_{i} \mid x_{k}\right)\right\}$ is the parameter of Bernoulli RFS. Since the $\Theta_{k, i}\left(x_{k}\right), i=1,2$ are independent, the probability mass of RFS $\Theta_{k}\left(x_{k}\right)$ is Eq. (8). 


$$
\begin{aligned}
\beta_{k}\left(S \mid x_{k}\right)=\prod_{i=1}^{2} \beta_{k, i}\left(S \mid x_{k}\right) \\
=\prod_{i=1}^{2}\left(1-p_{D, k}^{i}\left(x_{k}\right)+p_{D, k}^{i}\left(x_{k}\right) p_{Y_{k, i}}\left(S \mid x_{k}\right)\right) \\
=\left(1-p_{D, k}^{1}\left(x_{k}\right)\right)\left(1-p_{D, k}^{2}\left(x_{k}\right)\right)+\left(1-p_{D, k}^{1}\left(x_{k}\right)\right) p_{D, k}^{2}\left(x_{k}\right) \\
\quad \int_{S} g_{k, 2}\left(y_{2} \mid x_{k}\right) d y_{2}+p_{D, k}^{1}\left(x_{k}\right)\left(1-p_{D, k}^{2}\left(x_{k}\right)\right) \int_{S} g_{k, 1}\left(y_{1} \mid x_{k}\right) d y_{1} \\
\quad+\frac{1}{2 !} p_{D, k}^{1}\left(x_{k}\right) p_{D, k}^{2}\left(x_{k}\right) \int_{S \times S} g_{k, 1}\left(y_{1} \mid x_{k}\right) g_{k, 2}\left(y_{2} \mid x_{k}\right) d y_{1} d y_{2}
\end{aligned}
$$

Thus, comparing Eq. (8) with the function of set integral, we can get the probability density of $\Theta_{k}\left(x_{k}\right)$ in Eq. (9).

$$
\pi_{\Theta_{k}}\left(Y_{k} \mid x_{k}\right)= \begin{cases}\left(1-p_{D, k}^{1}\left(x_{k}\right)\right)\left(1-p_{D, k}^{2}\left(x_{k}\right)\right) & \text { if } Y_{k}=\varnothing \\ p_{D, k}^{1}\left(x_{k}\right)\left(1-p_{D, k}^{2}\left(x_{k}\right)\right) g_{k, 1}\left(y_{1} \mid x_{k}\right) & \text { if } Y_{k}=\left\{y_{1}\right\} \\ \left(1-p_{D, k}^{1}\left(x_{k}\right)\right) p_{D, k}^{2}\left(x_{k}\right) g_{k, 2}\left(y_{2} \mid x_{k}\right) & \text { if } Y_{k}=\left\{y_{2}\right\} \\ \left.p_{D, k}^{1}\left(x_{k}\right) p_{D, k}^{2}\left(x_{k}\right) g_{k, i}\left(y_{a} \mid x_{k}\right) g_{k, j}\left(y_{b} \mid x_{k}\right)\right) & \text { if } Y_{k}=\left\{y_{1}, y_{2}\right\}\end{cases}
$$

From the RFS model in Eq. (5), we know $Y=\Theta_{k}\left(x_{k}\right) \bigcup W_{k, 1} \bigcup L \bigcup W_{k, n}$. Thus, substituting the Eq. (9) into the fundamental convolution formula [1, Sec.11.5], the probability density of the state $x_{k}$ at time $k$ produces the measurements set $Y_{k}$ can be obtained in Eq. (10).

$$
\begin{aligned}
\eta\left(Y_{k} \mid x_{k}\right)=\pi_{\Theta_{k}}\left(\varnothing \mid x_{k}\right) K\left(Y_{k}\right)+\sum_{y_{1} \in Y_{k, 1}} \pi_{\Theta_{k}}\left(\left\{y_{1}\right\} \mid x_{k}\right) K\left(Y_{k}-\left\{y_{1}\right\}\right) \\
\quad+\sum_{y_{2} \in Y_{k, 2}} \pi_{\Theta_{k}}\left(\left\{y_{2}\right\} \mid x_{k}\right) K\left(Y_{k}-\left\{y_{2}\right\}\right)+\sum_{\left\{y_{1}, y_{2}\right\} Y_{k}} \pi_{\Theta_{k}}\left(\left\{y_{1}, y_{2}\right\} \mid x_{k}\right) K\left(Y_{k}-\left\{y_{1}, y_{2}\right\}\right) \\
=\left(1-p_{D, k}^{1}\left(x_{k}\right)\right)\left(1-p_{D, k}^{2}\left(x_{k}\right)\right) K_{1}\left(Y_{k, 1}\right) K_{2}\left(Y_{k, 2}\right) \\
\quad+\sum_{y_{1} \in Y_{k}} p_{D, k}^{1}\left(x_{k}\right)\left(1-p_{D, k}^{2}\left(x_{k}\right)\right) g_{k, 1}\left(y_{1} \mid x_{k}\right) K_{1}\left(Y_{k, 1}-\left\{y_{1}\right\}\right) K_{2}\left(Y_{k, 2}\right) \\
\quad+\sum_{y_{1} \in Y_{K}}\left(1-p_{D, k}^{1}\left(x_{k}\right)\right) p_{D, k}^{2}\left(x_{k}\right) g_{k, 2}\left(y_{2} \mid x_{k}\right) K_{1}\left(Y_{k, 1}\right) K_{2}\left(Y_{k, 2}-\left\{y_{2}\right\}\right) \\
\left.\quad+\sum_{\left\{y_{1}, y_{2}\right\} \in Y_{k}} p_{D, k}^{1}\left(x_{k}\right) p_{D, k}^{2}\left(x_{k}\right) g_{k, 1}\left(y_{1} \mid x_{k}\right) g_{k, 2}\left(y_{2} \mid x_{k}\right)\right) K_{1}\left(Y_{k, 1}-\left\{y_{1}\right\}\right) K_{2}\left(Y_{k, 2}-\left\{y_{2}\right\}\right)
\end{aligned}
$$

Since the Bernoulli filter has no closed form solution, the SMC method is applied to implement the MSBF, which is similar to the method used in [5] (further details, see [5]).

\section{Numerical Simulations}

\subsection{Scenario}

This section presents a single target tracking example to compare the SMC-MSBF's performance with the traditional SMC-MSBF via numerical simulations.

The target state is $x_{k}=\left[p_{x, k}, \&_{x, k}, p_{y, k}, \&_{y, k}\right]^{T}$, where $\left(p_{x, k}, \&_{x, k}\right)$ and $\left(p_{y, k}, \&_{y, k}\right)$ denote the target position and velocity. The target dynamic is modeled by

$$
x_{k}=\left[\begin{array}{cccc}
1 & T & 0 & 0 \\
0 & 1 & 0 & 0 \\
0 & 0 & 1 & T \\
0 & 0 & 0 & 1
\end{array}\right] x_{k-1}+v_{k}
$$

Where $T$ is the sampling interval and $v_{k}$ is zero mean white Gaussian noise with the covariance $Q$ 


$$
Q=\left[\begin{array}{cccc}
\frac{T^{3}}{3} & \frac{T^{2}}{2} & 0 & 0 \\
\frac{T^{2}}{2} & T & 0 & 0 \\
0 & 0 & \frac{T^{3}}{3} & \frac{T^{2}}{2} \\
0 & 0 & \frac{T^{2}}{2} & T
\end{array}\right] \sigma_{v}^{2}
$$

In the tracking scenario, we assume that one target appears at scan $k=1$ and disappears at scan $k$ $=60$ with the initial target state $x_{0}=(550 \mathrm{~m},-5 \mathrm{~m} / \mathrm{s}, 300 \mathrm{~m},-8.5 \mathrm{~m} / \mathrm{s})$, the sampling interval is $T=1 \mathrm{~s}$ and $\sigma_{v}^{2}=0.05 \mathrm{~m}^{2} \mathrm{~s}^{-3}$. There are two sensors in this tracking scenario, which located at $p_{S_{1}}=[0 ; 0]$ and $p_{S_{2}}=[100 ; 800]$ respectively.

This scenario uses range and azimuth measurements and defined as:

$$
y_{S_{i}, k}=\left[\begin{array}{c}
\sqrt{\left(p_{x, k}-p_{S_{i}, x}\right)^{2}+\left(p_{y, k}-p_{S_{i}, y}\right)^{2}} \\
\arctan \left(\frac{p_{x, k}-p_{S_{i}, x}}{p_{y, k}-p_{S_{i}, y}}\right)
\end{array}\right]+w_{k}
$$

Where $\left[p_{s_{i}, x}, p_{S_{i}, y}\right]^{T}$ denotes the location of sensor $i$, and $w_{k}$ is zero mean white Gaussian with covariance $\sum=\operatorname{diag}\left[\sigma_{r}^{2}, \sigma_{\theta}^{2}\right]\left(\sigma_{r}=2.5 \mathrm{~m}\right.$ and $\left.\sigma_{\theta}=0.25^{\circ}\right)$. Clutter is modeled as Poisson RFS with the average number of $\lambda=5$ returns per scan. The surveillance region is the half disc $[-\pi / 2, \pi / 2]$ $\operatorname{rad} \times[0,1500] \mathrm{m}$, each sensor has the same reentry probability and target survive probability that are set to $p_{R, k}=0.01$ and $p_{S, k}=0.95$. We evaluate the performance using the optimal subpattern assignment (OSPA) metric (further details, see [8]).

\subsection{Simulation Results}

To evaluate the tracking performance of SMC-MSBF, we compare it with the traditional SMC-MSBF (the iterated-corrector approximation) over 100 Monte Carlo trials through two examples: the same detection probability and the different detection probability between two sensors, and the OSPA parameters are set to cutoff parameter $c=25 \mathrm{~m}$ and order parameter $p=1$.

1) The average of OSPA distance for same detection probability with two sensors (both $p_{D}=0.7$ ) is shown in fig. 1 . The simulation results demonstrate that the SMC-MSBF provides more accurate position estimates than the traditional algorithm.

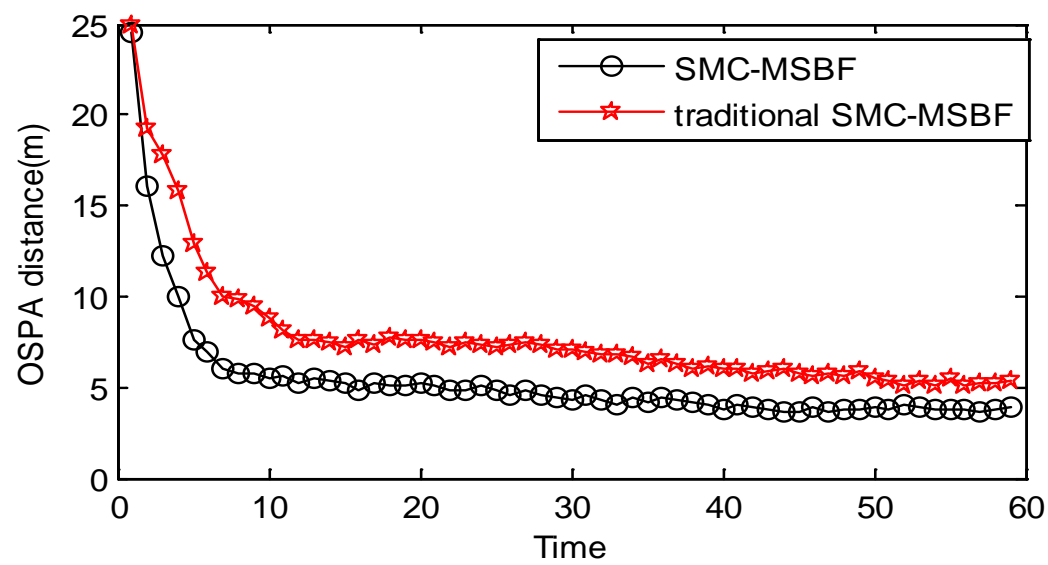

Fig. 1 Average of OSPA distance (the same detection probability with two sensors) 
2) The detection probability of two sensors are $p_{D}=0.5$ and $p_{D}=0.8$ respectively. Due to the traditional SMC-MSBF based on the 'iterated-corrector approximation', we change the order of two sensors to evaluate the algorithms. The traditional SMC-MSBF include two cases: detection probability of last sensor $p_{D}=0.5$ or $p_{D}=0.8$. The average of OSPA distance for the different detection probability with two sensors is shown in fig. 2. The simulation results shows that the performance of SMC-MSBF is surpasses the performance of the traditional SMC-MSBF. Its OSPA distance is smaller than the traditional SMC-MSBF. Especially, when the detection probability of the last sensor is relatively low (the detection probability of last sensor $p_{D}=0.5$ ) in the traditional algorithm, the performance of proposed SMC-MSPF is more extraordinary. This is because the performance of traditional SMC-MSBF largely depends on the detection probability of the last sensor. When the detection probability of the last sensor is low, the target may often be undetected (all the measurements from this sensor are clutter), and using this measurements and parameters of the last sensor to update the Bernoulli RFS will produce a relatively large error. However, the proposed SMC-MSBF use the measurements of all the sensors at the same time to track the target, which is theoretically superior to the traditional SMC-MSBF. Also the performance of the proposed algorithm is invariant under sensor reordering.

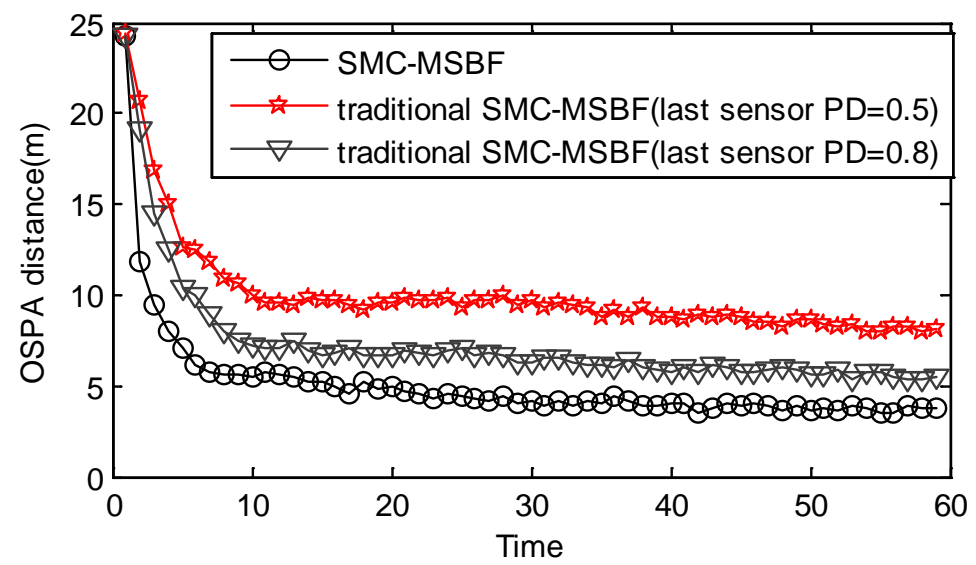

Fig. 2 Average of OSPA distance (the different detection probability with two sensors)

\section{Conclusion}

In this paper, the MSBF, which tracks a single target using multi-sensor, has been proposed. The proposed MSBF is based on the theory of FISST, the FISST is applied to derive the multi-sensor likelihood function, and the SMC method is used to implement the MSBF algorithm. Simulation results show that the proposed MSBF can track a single target effectively, and the proposed MSBF shows excellent performance compared with the traditional MSBF.

\section{References}

[1]. R. Mahler, Statistical Multisource Multitarget Information Fusion. Norwood, MA, USA: Artech House, 2007.

[2]. R. P. S.Mahler. Multi-target Bayes filtering via first-ordermulti-target moments. IEEE Transactions on Aerospace and Electronic Systems. vol. 39 (2003). No. 4, p. 1152-1178.

[3]. R. P. S. Mahler. PHD filters of higher order in target number. IEEE Transactions on Aerospace and Electronic Systems. vol. 43 (2007) No. 4, pp. 1523-1543.

[4]. B.-T. Vo, B. Vo, A. Cantoni. The cardinality balanced multi-target multi-Bernoulli filter and its implementations. IEEE Transactions on Signal Processing. vol. 57 (2009), No. 2, p. 409-423.

[5]. B.-T. Vo, D. Clark, B.-N. Vo, et al. Bernoulli forward-backward smoothing for joint target detection and tracking. IEEE Transactions on Signal Processing. vol. 59 (2011) No. 9, p. 4473-4477. 
[6]. B.-T. Vo, C.-M. See, N. Ma, et al. Multi-sensor joint detection and tracking with the Bernoulli filter. IEEE Transactions on Aerospace and Electronic Systems. vol. 48 (2012) no. 2. p. 1385-1402.

[7]. J Xu, F Huang, Z Huang. The multi-sensor PHD filter Analytic implementation via Gaussian mixture and effective binary partition. Proc. 16th International Conf. on Information Fusion, Istanbul, TUR, July 2013, p. 945-952.

[8]. Schuhmacher, D., Vo, B.-T., Vo, B.-N. A consistent metric for performance evaluation of multi-object filters. IEEE Transactions on Signal Processing. vol. 56 (2008), No. 8, p. 3447-3457. 